\title{
TERAPIA COMUNITÁRIA INTEGRATIVA COMO INSTRUMENTO DE INTEGRAÇÃO, EMPODERAMENTO E AUTOCUIDADO DE USUÁRIOS DE UMA ASSOCIAÇÃO DE DIABÉTICOS
}

\author{
TERAPIA COMUNITARIA INTEGRADORA COMO INSTRUMENTO DE \\ INTEGRACIÓN, EMPODERAMIENTO Y AUTOCUIDADO DE USUARIOS DE UNA \\ ASOCIACIÓN DE DIABÉTICOS
}

INTEGRATIVE COMMUNITY THERAPY AS AN INSTRUMENT OF INTEGRATION, EMPOWERMENT AND SELF-CARE FOR USERS OF A DIABETIC ASSOCIATION

\author{
Angelina VASCONCELLOS DE CHAZARRETA ${ }^{1}$ \\ Oscar KENJI NIHEI ${ }^{2}$ \\ Sâmia FERREIRA LEITE ${ }^{3}$ \\ Claudio MARLON DA SILVA ${ }^{4}$ \\ Hulyana PEREIRA PARDINHO 5 \\ Etielle Talita RIBEIRO AGUIAR ${ }^{6}$ \\ Terezinha ZAGOTTA MACHADO PINEZI ${ }^{7}$ \\ Walfrido KÜHL SVOBODA ${ }^{8}$
}

RESUMO: O estudo objetivou analisar os possíveis efeitos da Terapia Comunitária Integrativa (TCI) na integração, empoderamento e autocuidado do controle do diabetes mellitus dos usuários da Associação de Diabéticos de Foz do Iguaçu-PR (ADIFI). Pesquisa descritiva e de delineamento quase-experimental. Na qual analisou-se os efeitos da TCI sobre o autocuidado

\footnotetext{
${ }^{1}$ Associação dos Diabéticos de Foz do Iguaçu (ADIFI), Foz do Iguaçu - PR - Brasil. Responsável Técnica da ADIFI. Graduação em Psicologia (UNIVALE). ORCID: https://orcid.org/0000-0002-5768-3012. E-mail: clodomira2002@hotmail.com

${ }^{2}$ Universidade Estadual do Oeste do Paraná (UNIOESTE), Foz do Iguaçu - PR - Brasil. Professor Associado do Centro de Educação, Letras e Saúde. Doutorado em Ciências (UFRJ). ORCID: https://orcid.org/0000-0002-91567787. E-mail: oknihei@gmail.com

${ }^{3}$ Universidade Federal da Integração Latino-Americana (UNILA), Instituto Latino-Americano de Ciências da Vida e da Natureza (ILACVN), Foz do Iguaçu - PR - Brasil. Graduanda do curso de Saúde Coletiva. ORCID: https://orcid.org/0000-0003-2274-3780. E-mail: samiaferrleite@gmail.com

${ }^{4}$ Universidade Estadual do Oeste do Paraná (UNIOESTE), Foz do Iguaçu - PR - Brasil. Especialização em andamento em Intervenção Breve em Psicologia. ORCID: https://orcid.org/0000-0001-9558-6232. E-mail: marlonfoz.2009@gmail.com

${ }^{5}$ Associação dos Diabéticos de Foz do Iguaçu (ADIFI), Foz do Iguaçu - PR - Brasil. Enfermeira na ADIFI. ORCID: https://orcid.org/0000-0001-6462-9498.E-mail: huly_ana@hotmail.com

${ }^{6}$ Associação dos Diabéticos de Foz do Iguaçu (ADIFI), Foz do Iguaçu - PR - Brasil. Nutricionista de Produção na ADIFI. Graduação em Nutrição (UNIAMERICA). ORCID: https://orcid.org/0000-0002-1457-5505. E-mail: etielleconsultoria@gmail.com

${ }^{7}$ Associação dos Diabéticos de Foz do Iguaçu (ADIFI), Foz do Iguaçu - PR - Brasil. Presidente da ADIFI. Graduação em Pedagogia (UNOESTE). ORCID: https://orcid.org/0000-0002-2369-2922. E-mail: pinezzi@hotmail.com

${ }^{8}$ Universidade Federal da Integração Latino-Americana (UNILA), Instituto Latino-Americano de Ciências da Vida e da Natureza (ILACVN), Foz do Iguaçu - PR - Brasil. Professor Associado do Curso de Saúde Coletiva, do Programa de Pós-Graduação em Políticas Públicas e Desenvolvimento e do Programa de Residência Multiprofissional em Saúde da Família. Doutorado em Ciência Animal (UEL). ORCID: https://orcid.org/00000001-6320-4754. E-mail: walfrido.svoboda@unila.edu.br
} 
dos participantes, e sobre parâmetros clínicos (glicemia, pressão arterial), antes e após um intervalo consecutivo de rodas de TCI. Aplicou-se o teste t pareado e regressão linear. Os encontros ocorreram entre 15/05/19 a 11/03/20. Constatou-se melhora da glicemia e pressão sistólica, possivelmente consequência de mudanças de hábitos dos participantes indicando maior autocuidado para o enfrentamento dessa doença crônica. Infere-se que a educação em saúde, desenvolvidos na ADIFI, pode também estar influenciando positivamente a qualidade de vida dos participantes. Conclui-se que os encontros de TCI auxiliaram na aquisição de maior resiliência, autocuidado e integração dos participantes.

PALAVRAS-CHAVE: Terapia comunitária integrativa. Diabetes. Tecnologia leve. Autocuidado. Empoderamento.

RESUMEN: El estudio tuvo como objetivo analizar los posibles efectos de la Terapia Integrativa Comunitaria (TIC) en el autocuidado del control de la diabetes mellitus de usuarios de la Asociación de Diabéticos de Foz do Iguaçu-PR (ADIFI). Investigación descriptiva y diseño cuasiexperimental. Se analizaron los efectos de las TIC sobre el autocuidado de los participantes y sobre los parámetros clínicos (glucemia, presión arterial), antes y después de un intervalo consecutivo de ruedas TIC. Se aplicó la prueba t pareada y la regresión lineal. Periodo de investigación: 15/05/19 y 03/11/20. Hubo mejora en la glucemia y la presión sistólica, posiblemente consecuencia de cambios en los hábitos que indican mayor autocuidado. Se infiere que la educación para la salud, desarrollada en ADIFI, también puede estar influyendo positivamente en la calidad de vida de los participantes. Se concluye que la TIC contribuyó con mayor resiliencia e integración de los participantes.

PALABRAS CLAVE: Terapia comunitaria integradora. Diabetes. Tecnología leve. Cuidados personales. Empoderamiento.

ABSTRACT: The study aimed to analyze the possible effects of the Integrative Community Therapy (ICT) on the self-care of users of Diabetic Association of Foz do Iguaçu-PR (ADIFI) to control diabetes mellitus. Descriptive and quasi-experimental design research. Where the effects of ICT were analyzed on the participants' self-care and on their clinical parameters (glycemia, blood pressure), before and after a consecutive interval of ICT circles. The paired $t$-test and linear regression were applied. Research period: 15/05/19 to 11/03/20. As a result, there was an improvement in glycemia and systolic pressure, possibly a consequence of changes in the participants' habits, indicating greater self-care for coping with this chronic disease. It is inferred that health education, developed at ADIFI, may also be positively influencing their quality of life. It is concluded that the ICT reunions helped in the acquisition of greater resilience, self-care and integration of the participants.

KEYWORDS: Integrative community therapy. Diabetes. Soft technology. Self-care. Empowerment. 


\section{Introdução}

A Terapia Comunitária Integrativa (TCI) foi proposta e criada pelo Prof. Dr. Adalberto Barreto em 1987, no município de Fortaleza-CE, com a intenção de solucionar as necessidades de saúde daquela comunidade (BARRETO, 2008).

As tecnologias de assistência em saúde podem ser classificadas em três categorias: (1) Dura, relacionada a equipamentos tecnológicos, normas, rotinas e estruturas organizacionais; (2) Leve-dura, que compreende todos os saberes bem estruturados no processo de saúde; e (3) Leve, que se refere às tecnologias de relações, de produção de comunicação, de acolhimento, de vínculos, de autonomização. Embora essas três categorias de tecnologias da saúde se interrelacionem, o ser humano necessita, em especial, das tecnologias de relações, definidas como 'leves' que são capazes de propiciar o acolhimento necessário para que cliente e profissional de saúde possam se beneficiar deste momento (MERHY; ONOCKO, 1997).

Considerando a complexidade do ser humano, o sujeito é contextualizado, estando seu estado de saúde dependente das condições ambientais, biológicas, psicológicas, do seu estilo de vida e das instituições em que se opera o cuidado. A conjunção desses fatores interfere nas tecnologias incorporadas à saúde. Desta forma, a Terapia Comunitária Integrativa (TCI) mostra sua relevância como tecnologia leve de cuidado em saúde mental, de baixo custo e de maior acessibilidade à população que focaliza de forma inovadora na reorganização das redes de atenção à saúde, em especial da atenção primária à saúde (ANDRADE et al., 2009).

A TCI é considerada uma ferramenta de construção de redes sociais solidárias, nas quais todos se tornam corresponsáveis na busca de soluções e superação dos desafios do cotidiano, num ambiente acolhedor e caloroso. Tem como base de sustentação o estímulo para a construção de vínculos solidários e promoção da vida. É um espaço comunitário onde se procura partilhar experiências de vida e sabedorias. Além disso, cria vínculos, resgata a autonomia dos indivíduos e ressignifica momentos de dores e perdas (BARRETO, 2008).

No ano de 2006 foi aprovada a Política Nacional de Práticas Integrativas e Complementares (PNPIC) no Sistema Único de Saúde do Ministério da Saúde, porém a Terapia Comunitária Integrativa não havia sido incorporada oficialmente a ela (BRASIL, 2006b). Nove anos mais tarde, em 2017, a TCI foi incluída na Política Nacional de Práticas Integrativas e Complementares (PNPIC), inserindo-a nos serviços de saúde, ampliando e reafirmando assim a visão do sistema oficial a respeito da saúde e autonomia das pessoas no autocuidado (BRASIL, 2017). 
O diabetes mellitus configura-se hoje como uma epidemia mundial, traduzindo-se em grande desafio para os sistemas de saúde de todo o mundo. O envelhecimento da população, a urbanização crescente e a adoção de estilos de vida pouco saudáveis como sedentarismo, dieta inadequada e obesidade são os grandes responsáveis pelo aumento da incidência e prevalência do diabetes em todo o mundo. Um indicador macroeconômico a ser considerado é que o diabetes cresce mais rapidamente em países pobres e em desenvolvimento, e isso impacta de forma muito negativa devido à morbimortalidade precoce que atinge pessoas ainda em plena vida produtiva, onera a previdência social e contribui para a continuidade do ciclo vicioso da pobreza e da exclusão social (BRASIL, 2006a).

As consequências humanas, sociais e econômicas são devastadoras: são 4 milhões de mortes por ano relativas ao diabetes e suas complicações, com muitas ocorrências prematuras, o que representa $9 \%$ da mortalidade mundial total. O grande impacto econômico ocorre notadamente nos serviços de saúde, como consequência dos crescentes custos do tratamento da doença e, sobretudo das complicações que podem surgir nos diabéticos, como a doença cardiovascular, a diálise por insuficiência renal crônica e as cirurgias para amputações de membros inferiores (SOUZA et al., 2012).

O maior custo, entretanto, recai sobre os portadores, suas famílias, seus amigos e comunidade: o impacto na redução de expectativa e qualidade de vida é considerável. A expectativa de vida é reduzida em média em 15 anos para o diabetes tipo I e em 5 a 7 anos na do tipo II. Adultos com diabetes têm risco 2 a 4 vezes maior de doença cardiovascular e acidente vascular cerebral. A diabetes é, ainda, a causa mais comum de amputações não traumáticas de membros inferiores, cegueira irreversível e doença renal crônica terminal. Em mulheres, é responsável por maior número de partos prematuros e mortalidade materna (BRASIL, 2006a).

Segundo a American Diabetes Association (1987) recomendações para o controle do diabetes mellitus (DM) tipos I e II incluem automonitorização de: (1) glicemia capilar; (2) medicação oral (quando necessária); (3) múltiplas doses de insulina (quando necessário), (4) alterações nos padrões dietéticos a partir de reeducação alimentar, (5) realização de atividades físicas programadas e (6) educação em diabetes permanente e continuada, a fim de manter os níveis glicêmicos. Estas recomendações implicaram mudanças de comportamento dos diabéticos e seus familiares (AMERICAN DIABETES ASSOCIATION, 1987).

Na prática interprofissional da Associação dos Diabéticos de Foz do Iguaçu-PR (ADIFI) foi constatado que fatores psicossociais como idade, crenças, valores, motivação pessoal, condições financeiras, resiliência, empoderamento e doenças associadas, entre outros aspectos, podem influenciar na decisão do diabético ou da família em optar por um controle mais rígido. 
Visando promover uma melhor qualidade de vida dos usuários diabéticos da ADIFI, a TCI foi implantada na Associação dos Diabéticos de Foz do Iguaçu (ADIFI) no ano de 2015 e continua atendendo aos usuários desde então. No entanto, uma pesquisa mais aprofundada da prática e potencial impacto da TCI entre diabéticos não havia ainda sido realizada.

Por esse motivo, o objetivo principal deste trabalho foi analisar os possíveis efeitos e impactos dos encontros de TCI na integração, empoderamento e autocuidado do controle do diabetes mellitus dos usuários da ADIFI.

\section{Metodologia}

\section{Delineamento do estudo}

Trata-se de um estudo descritivo e quase experimental. $\mathrm{O}$ delineamento quase experimental é aquele onde procura-se uma relação entre uma variável dependente e a independente, manipulando-se a variável independente, mas sem recorrer a recursos de aleatoriedade da amostra e um grupo controle puro (BAPTISTA; CAMPOS, 2007). Na presente pesquisa, optou-se por uma análise do tipo pré-teste e pós-teste, onde os mesmos sujeitos foram analisados antes e após a realização de um número determinado de intervenções, de forma que os participantes foram os controles de si mesmos.

A intervenção aplicada foi a TCI, que se baseou na metodologia proposta pelo Prof. Dr. Adalberto Barreto, precursor da proposta tanto a nível nacional como mundial (BARRETO, 2010). Tal metodologia tem como fundamentos teóricos cinco grandes eixos, a saber: 1) pensamento sistêmico; 2) teoria da comunicação; 3) antropologia cultural; 4) pedagogia de Paulo Freire e 5) resiliência.

\section{Caracterização da população-alvo e o local do estudo}

O estudo foi realizado na ADIFI, uma Associação Civil de saúde que tem como objetivo promover Educação em Diabetes, difundir experiências e conhecimentos aos seus usuários, sobretudo educação continuada, como principal forma de prevenção, qualidade de vida e atendimento aos seus direitos sócio assistenciais. Sempre buscando acompanhar as inovações e pesquisas científicas no enfrentamento da doença, de forma planejada, permanente e continuada, bem como, desenvolver diferentes tipos de projetos que visem melhoria da qualidade de vida de seus associados. 
A população do estudo foi constituída por diabéticos, usuários da ADIFI, participantes dos encontros de TCI. Os participantes eram de ambos os sexos, sem limite de idade, mas todos com idade superior a 18 anos idade e portadores de diabetes mellitus tipo I ou II e totalizaram 21 participantes. A referida Associação se localiza na região de Tríplice Fronteira Internacional (Brasil-Paraguai-Argentina), no município de Foz do Iguaçu-PR.

\section{Instrumento e coleta dos dados}

Aplicou-se um instrumento semiestruturado, que continha questões relativas ao perfil sócio demográfico, relato de experiências compartilhadas no coletivo e às auto percepções dos participantes sobre as atividades propostas. Sendo que cada roda de TCI gerou um relatório final do encontro, e as informações relativas aos encontros de TCI foram extraídas desses documentos. Os instrumentos estruturados utilizados para determinar o autocontrole e empoderamento no tratamento contínuo da diabetes mellitus foram a aferição de glicemia capilar e pressão arterial que eram disponibilizadas aos usuários que participaram da pesquisa (sujeitos de pesquisa), sempre antes do início de todos os encontros de TCI. A finalidade foi verificar se houve alguma mudança nos níveis da glicemia capilar e pressão arterial após a participação em um número determinado de Rodas de TCI, e como esses níveis se apresentaram após o término da pesquisa.

\section{Os encontros de TCI}

Os encontros de TCI foram realizadas na modalidade presencial, em $100 \%$ dos casos, entre 15/05/2019 e 11/03/2020, às quartas-feiras, no horário vespertino das 14:00 às 16:30h. Na etapa de aplicação prática de cada encontro de TCI, foram observadas e analisadas cinco fases. Cabe ressaltar que, cada encontro de TCI constituiu-se como processo terapêutico completo com momento inicial, médio e final (BARRETO, 2008). os encontros de TCI foram conduzidas por um terapeuta e um coterapeuta convidado, a partir de situações/problema trazidas por um ou mais integrantes da roda.

As cinco etapas da intervenção em TCI foram seguidas de acordo com a descrição contida no livro "Terapia Comunitária Integrativa: passo a passo", de Adalberto Barreto (BARRETO, 2008), que foram: acolhimento, escolha do tema, contextualização, problematização, finalização. 
Vale informar que foi preconizado o uso da linguagem popular em todas as fases dos encontros de TCI, ou seja, aquela que não precisa de termos/conceitos técnicos para a correta abordagem dos cenários propostos. Desta forma, foi adotada uma comunicação assertiva, que não acarretasse coibição nas etapas de recepção, análise e transmissão das informações, em quaisquer das partes (participantes e pesquisadores), sendo esse um comportamento esperado do terapeuta e dos voluntários da TCI.

\section{Análise e tratamento dos dados}

Os dados obtidos por meio do instrumento semiestruturado foram tabulados em planilhas do programa Excel (Microsoft, 2010, EUA) e a análise estatística foi realizada utilizando-se o programa Minitab ${ }^{\mathrm{TM}} 18.1$ (2017, EUA). Foi aplicada a estatística descritiva para a obtenção do número absoluto, percentual, média e desvio padrão dos resultados. Para comparação dos parâmetros clínicos (glicemia, pressão sistólica e diastólica) dos participantes entre intervalo de 5 a 6 encontros, primeiramente aplicou-se o teste de normalidade Kolmogorov-Smirnov, que indicou que os dados apresentavam distribuição normal. Em seguida, foi aplicado o teste t pareado, com nível de significância de 5\%, para se comparar os resultados do início da participação (pré-teste) com a do final (pós-teste) desse intervalo total. Para verificar a influência da quantidade de rodas de TCI participadas (variável independente) sobre a média das variáveis clínicas (variáveis dependentes contínuas) dos participantes (glicemia, pressão sistólica e diastólica), aplicou-se a regressão linear, com nível de significância de $5 \%$. Todas as análises foram realizadas utilizando-se o programa Minitab ${ }^{\mathrm{TM}}$ 18.1 (2017, EUA).

$\mathrm{Na}$ leitura integral dos relatórios finais dos encontros de TCI, surgiram duas categorias para a análise do discurso "temas universais" e "estratégias de enfrentamento". Nos temas universais foi ressaltado o grau de reiteração, ou seja, quantas vezes uma temática específica foi mencionada pelos participantes. Por sua vez, os temas universais foram agrupados em quatro principais eixos, a saber: (1) problemas físicos e psíquicos de saúde; (2) conflitos familiares; (3) morte, perdas e/ou angústias; (4) suicídio.

Para as estratégias de enfrentamento foi empregada à mesma técnica de análise. A agrupamento desta categoria constituiu-se em quatro eixos, a saber: (1) fortalecimento e empoderamento pessoal; (2) busca de ordem espiritual e/ou religiosa; (3) busca de redes solidárias e (4) cuidado do relacionamento com a família e autocuidado. 


\section{Aspectos éticos}

Este trabalho acatou os princípios bioéticos da pesquisa em saúde com seres humanos conforme a resolução CNS 466/2012. Após esclarecimento sobre os riscos e benefícios possíveis decorrentes da pesquisa, obteve-se assinatura do Termo de Compromisso Livre e Esclarecido (TCLE), por parte dos participantes (BRASIL, 2012).

\section{Resultados e Discussão}

Neste estudo participaram 21 usuários da ADIFI, ao longo de quatorze encontros presenciais. A Tabela 1 apresenta o perfil sócio demográfico dos participantes dos encontros de TCI realizados na ADIFI, contendo: sexo, faixa etária, escolaridade, estado civil, religião e ocupação.

Pode-se observar a partir da Tabela 1, que o grupo foi composto na maioria por homens $(57,1 \%)$, mas também o número de mulheres foi significativo; o que indica que houve uma boa adesão de usuários de ambos os sexos na terapia. Outro fato identificado foi referente à faixa etária, que variou de 50 anos até mais de 75 anos de idade; sendo que a maioria se encontrava entre 70 anos a 74 anos de idade (38,1\%). Quanto ao estado civil, religião e ocupação, a maioria dos participantes se declararam casados, de religião evangélica e aposentados. Um dado relevante é que o grupo alcança todas as escolaridades, sendo assim um grupo formado por integrantes de diferentes graus de formação.

Tabela 1 - Perfil sócio demográfico dos participantes dos encontros de TCI realizados na ADIFI, Foz do Iguaçu-PR, Brasil, 2019/2020

\begin{tabular}{lc}
\hline Variável & $\mathbf{N}(\mathbf{\%})$ \\
\hline Sexo & \\
Feminino & $9(42,9)$ \\
Masculino & $12(57,1)$ \\
\hline Faixa Etária & \\
50 a 59 anos & $5(23,8)$ \\
60 a 69 anos & $6(28,6)$ \\
70 a 74 anos & $8(38,1)$ \\
$\geq 75$ anos & $2(9,5)$ \\
\hline Escolaridade & $11(52,4)$ \\
Fundamental & $5(23,8)$ \\
Médio & $5(23,8)$ \\
Superior & \\
\hline Estado Civil & $13(61,9)$ \\
Casado & \\
\hline
\end{tabular}




\begin{tabular}{lc}
\hline Solteiro & $2(9,5)$ \\
Separado & $3(14,3)$ \\
Viúvo & $3(14,3)$ \\
\hline Religião & \\
Católico & $8(38,1)$ \\
Evangélico & $10(47,6)$ \\
Outro & $2(9,5)$ \\
Não tem & $1(4,8)$ \\
\hline Ocupação (resposta múltipla) & \\
Aposentado/Benefícios & $18(85,7)$ \\
Autônomo & $4(19,0)$ \\
Do Lar & $1(4,7)$ \\
\hline Total & $\mathbf{2 1 ( \mathbf { 1 0 0 } )}$ \\
\hline
\end{tabular}

Fonte: elaborado pelos autores

Conforme indicado na Tabela 2, pode-se observar que a maioria do grupo declarou que realiza atividade física, dorme bem, têm vínculos familiares, não é tabagista, não é etilista, não possuem comorbidades, são usuários do SUS, antes não haviam participado de Terapia Comunitária e não haviam se submetidos a outras práticas complementares de saúde. Foi observado ainda que $23,8 \%$ dos participantes assumiram ser alcoolistas, e entre as comorbidades citadas pelo grupo, de maior ocorrência, foram relatados os problemas cardíacos.

Tabela 2 - Perfil dos hábitos de vida, comorbidades e acesso ao sistema de saúde e terapias dos participantes dos encontros de TCI realizados na ADIFI, Foz do Iguaçu-PR, Brasil, $2019 / 2020$

\begin{tabular}{lc}
\hline Variável & $\mathbf{N}(\%)$ \\
\hline Realiza Atividade Física? & $11(52,4)$ \\
Sim & $10(47,6)$ \\
Não & $14(66,7)$ \\
\hline Dorme bem? & $7(33,3)$ \\
Sim & \\
Não & $18(85,7)$ \\
\hline Tem vínculos familiares? & $3(14,3)$ \\
Sim & \\
Não & $0(0,0)$ \\
\hline Tabagismo & $21(100,0)$ \\
Sim & \\
Não & $5(23,8)$ \\
\hline Etilismo & $16(76,2)$ \\
Sim & \\
Não & $5(23,8)$ \\
\hline Comorbidades (resposta múltipla) & $3(14,3)$ \\
Problemas cardíacos & $2(9,5)$ \\
Problemas Circulatórios (Vascular) & $11(52,4)$ \\
Transtornos Mentais & \\
Nenhum & $15(71,4)$ \\
\hline Atendimento Médico (resposta múltipla) & $8(38,1)$ \\
Sistema Público (SUS) & \\
Sistema Privado (Plano de Saúde) & \\
\hline
\end{tabular}




\begin{tabular}{lc}
\hline Participou de terapia Comunitária antes? & \\
Sim & $4(19,1)$ \\
Não & $17(80,9)$ \\
\hline Submeteu-se a outras práticas complementares de & \\
saúde? & $6(28,6)$ \\
Sim & $15(71,4)$ \\
Não & $\mathbf{2 1 ( 1 0 0 )}$ \\
\hline Total
\end{tabular}

Fonte: elaborado pelos autores

Quanto aos temas universais, que foram os problemas trazidos pelo grupo, os de maior ocorrência foram de ordem física e psíquica $(35,7 \%)$, seguidos por conflitos familiares $(28,6 \%)$ e morte, perdas e/ou angústias (21,4\%). Nas estratégias de enfrentamento, o grupo apresentou como principais soluções para os problemas a busca de ordem espiritual ou religiosa $(71,4 \%)$; seguidos por resiliência e empoderamento pessoal $(35,7 \%)$ e cuidado com o relacionamento familiar (35,7\%) (Tabela 3). O autocuidado e responsabilidade para com o próprio corpo foi a estratégia menos adotada pelo grupo pesquisado $(7,1 \%)$.

Tabela 3 - Temas universais e estratégias de enfrentamento identificados pelos participantes dos encontros de TCI realizados na ADIFI, Foz do Iguaçu-PR, Brasil, 2019/2020

\begin{tabular}{lc}
\hline & N (\%) \\
\hline Temas Universais (Um por encontro) & \\
Problemas físicos e psíquicos de saúde & $5(35,7)$ \\
Conflitos familiares & $4(28,6)$ \\
Morte, perdas e/ou angústias & $3(21,4)$ \\
Suicídio & $2(14,3)$ \\
\hline
\end{tabular}

\begin{tabular}{lc}
\hline Estratégias de Enfrentamento (Múltipla) & $10(71,4)$ \\
Busca de ordem espiritual ou religiosa & $5(35,7)$ \\
Resiliência e empoderamento pessoal & $5(35,7)$ \\
Cuidado com o relacionamento familiar & $3(21,4)$ \\
Estabelecimento de redes solidárias & $1(7,1)$ \\
Autocuidado e responsabilidade para próprio corpo & $\mathbf{1 4}(\mathbf{1 0 0})$ \\
\hline Total de encontros
\end{tabular}

Fonte: elaborado pelos autores

No presente estudo, verificou-se se houve diferença nos parâmetros clínicos glicemia, pressão sistólica e pressão diastólica de 8 participantes que apresentaram assiduidade e frequência consecutiva entre 5 e 6 rodas de TCI. Por meio da comparação desses parâmetros dos participantes, entre a $1^{\mathrm{a}}$ e a $5^{\mathrm{a}} / 6^{\mathrm{a}}$ roda de TCI, contatou-se que a pressão sistólica se mostrou significativamente menor no final do período analisado $(\mathrm{p}<0,05)$ (Tabela 4$)$. 
Tabela 4 - Comparação de parâmetros clínicos (glicemia, pressão sistólica e diastólica) dos participantes no intervalo entre encontros de TCI consecutivos realizados na ADIFI, Foz do Iguaçu-PR, Brasil, 2019/2020

\begin{tabular}{llcl}
\hline Parâmetros* & $1^{\circ}$ Encontro** & $5^{*} / 6^{\circ}$ Encontro** & $p$ \\
\hline Glicemia $(\mathrm{mg} / \mathrm{dL})$ & $200,5(72,6)$ & $145,9(27,0)$ & 0,081 \\
Pressão Sistólica $(\mathrm{mmHg})$ & $142,5(32,0)$ & $133,8(22,6)$ & 0,380 \\
Pressão Diastólica $(\mathrm{mmHg})$ & $82,5(13,8)$ & $72,5(13,8)$ & $0,007 * * *$ \\
\hline
\end{tabular}

*Parâmetros de 8 participantes com participação mínima entre 5 e 6 rodas de TCI consecutivamente. **Média e desvio padrão; *** $\mathrm{p}<0,05$ (Teste t pareado).

Fonte: elaborado pelos autores

Visando aprofundar as análises, verificou-se a correlação entre a quantidade de rodas de TCI frequentadas (variável independente) e os parâmetros clínicos glicemia, pressão sistólica e pressão diastólica (variáveis dependentes contínuas) por meio da análise de regressão linear. Conforme indicado na Tabela 5, o parâmetro clínico glicemia apresentou coeficiente de regressão linear negativo e significativo em relação à quantidade de rodas frequentadas, indicando redução significativa da glicemia no final do período analisado.

Tabela 5 - Análise de regressão linear entre a média dos parâmetros clínicos (glicemia, pressão sistólica e diastólica) dos participantes e o número de encontros de TCI frequentados, realizados na ADIFI, Foz do Iguaçu-PR, Brasil, 2019/2020

\begin{tabular}{llcc}
\hline Parâmetros* & Coeficiente & IC $(95 \%)$ & $p^{* *}$ \\
\hline Glicemia (mg/dL) & $-14,3$ & $(-21,9 ;-6,7)$ & $\mathbf{0 , 0 0 6}$ \\
Pressão Sistólica (mmHg) & 1,2 & $(-4,2 ; 6,6)$ & 0,560 \\
Pressão Diastólica (mmHg) & $-0,9$ & $(-2,6 ; 0,6)$ & 0,172 \\
\hline *Parâmetros de 8 participantes; com participação mínima entre 5 e 6 rodas de conversa; **p>0,05. \\
Fonte: elaborado pelos autores
\end{tabular}

O delineamento quase experimental apresenta limitações implícitas ao método, além disso, não se pode garantir que outras variáveis possam estar influenciando os resultados obtidos, tais como hábitos de vida, autocuidado e alimentação, por exemplo, pelo fato do intervalo entre as medições ser grande ( 5 a 6 rodas de TCI), impossibilitando fazer qualquer afirmação de causalidade, no entanto, os resultados apresentados trazem evidências que sugerem que, no contexto pesquisado, os encontros de TCI acarretaram, direta ou indiretamente, mudanças que beneficiaram os participantes e seus parâmetros clínicos pressão sistólica e glicemia. Sendo necessários estudos futuros para aprofundar tais impactos da TCI no comportamento e saúde dos usuários. 


\section{Considerações finais}

Com os resultados obtidos, foi possível constatar que a utilização dos encontros de TCI serviu, de modo geral, como ferramenta para a melhoria na qualidade de vida e bem-estar de um grupo de diabéticos da ADIFI presente da região brasileira de tríplice fronteira (Brasil Paraguai-Argentina).

Os parâmetros analisados que apresentaram significância estatística (pressão diastólica e glicemia) podem ter sido influenciados pelas intervenções de TCI, mas não somente por estas, pois não se pode descartar a influência do trabalho educativo e de acompanhamento realizado pela ADIFI, beneficiando comportamentos e a saúde dos participantes, atuando concomitantemente à intervenção realizada. No entanto, os resultados sugerem que os encontros de TCI acarretaram resultados benéficos em relação a esses parâmetros.

É fundamental estabelecer estratégias que redimensionem o foco de atenção da doença para o indivíduo, na perspectiva de causalidade do adoecimento como um eixo composto por variáveis multifacetadas e integradas que, quando desestabilizadas, repercutem, diretamente, na saúde das pessoas e das comunidades. Verificou-se que, durante os encontros de TCI, usuários não apenas recebiam e se constituíam, mas também criavam, inovavam e se transformavam, ou seja, eram sujeitos ativos, que participaram e interviram em seus diferentes contextos sociais, de modo que os problemas e as dificuldades, somatizadas através do sofrimento e das doenças, foram compreendidos como produtos de uma interação multidimensional.

Com os resultados obtidos é possível inferir que a TCI contribuiu de forma direta e/ou indireta para o fomento do empoderamento e do fortalecimento de vínculos que tiveram como consequência a adesão ao tratamento através da educação em diabetes e a melhoria da qualidade de vida, práticas sempre presentes na ADIFI.

A aplicação da TCI durante o desenvolvimento desta pesquisa se mostrou um espaço terapêutico de fala, escuta e aprendizagem, que colaborou com o programa de educação em diabetes desenvolvido pela ADIFI. Desta forma, os resultados obtidos pela aplicação desta tecnologia social leve de cuidado não representaram uma linha de chegada, mas revelaram um ponto de partida para futuras investigações que permitirão novos desdobramentos de apoio aos diabéticos.

As atividades desenvolvidas nesta pesquisa estão diretamente relacionadas às propostas de inclusão e de reabilitação psicossocial dos usuários e à apropriação de conhecimentos compartilhados no grupo pela mediação do terapeuta comunitário dos saberes e experiências de 
cada um. Nesses encontros terapêuticos, as vivências emanadas dos participantes compõem um saber que leva à resiliência, à autonomia e ao empoderamento individual e coletivo. Nesse sentido, tentar dar um "desfecho" a longos meses de trabalho e de dedicação foi algo complexo. Acredita-se que uma pesquisa não se inicia configurada para ser concluída, ela representa o disparar de uma ideia emanada que possa iluminar futuras investigações. Este é o verdadeiro sentido do conhecimento: trazer à tona descobertas que possam ser refutadas e/ou aperfeiçoadas continuamente em uma realidade que se renova de maneira cada vez mais célere.

AGRADECIMENTOS: À Pró-Reitoria de Extensão (PROEX) da UNILA, pela concessão do auxílio financeiro no Projeto de Extensão Universitária "Integrando a UNILA com Rodas de Conversa"; À Prefeitura Municipal de Foz do Iguaçu que através da Secretaria Municipal de Saúde (SMSA) mantém convênio com a ADIFI e possibilita a continuidade dos serviços prestados pela instituição; À ADIFI pela disponibilização do espaço físico e dos equipamentos necessários para a realização dos encontros de TCI; Aos usuários, estagiários e equipe de trabalho da ADIFI, pela oportunidade de realizar as intervenções terapêuticas grupais e pelo compartilhamento das experiências de vida que tanto enriqueceram este trabalho.

\section{REFERÊNCIAS}

AMERICAN DIABETES ASSOCIATION. Diabetes Control and Complications Trial (DCCT): resultados do estudo de viabilidade. Grupo de pesquisa DCCT. Diabetes Care, v. 10, n. 1, p. 1-19. 1987. Disponível em: https://care.diabetesjournals.org/content/10/1/1. Acesso em: 15 jul. 2020.

ANDRADE, L. O. M. et al. (Org.) Ministério da Saúde. Departamento de Atenção Básica. O SUS e a Terapia Comunitária. Fortaleza: Ministério da Saúde, 2009.

BAPTISTA, M. N.; CAMPOS, D. C. Metodologias de pesquisa em ciências: análises quantitativa e qualitativa. Rio de Janeiro: LTC, 2007.

BARRETO, A. P. Terapia comunitária: passo a passo. 4. ed. Fortaleza: Gráfica LCR, 2008.

BRASIL. Ministério da Saúde. Secretaria de Atenção à Saúde. Departamento de Atenção Básica. Diabetes Mellitus. Brasília: Ministério da Saúde, 2006a. Disponível em: http://bvsms.saude.gov.br/bvs/publicacoes/diabetes_mellitus_cab16.pdf. Acesso em: 15 jul. 2020.

BRASIL. Portaria n. 971, de 03 de maio de 2006. Aprova a Política Nacional de Práticas Integrativas e Complementares (PNPIC) no Sistema Único de Saúde. Brasília: Ministério da Saúde, 2006b. Disponível em:

http://bvsms.saude.gov.br/bvs/saudelegis/gm/2006/prt0971_03_05_2006.html. Acesso em: 15 jul. 2020. 
BRASIL. Resolução n. 466, de 12 de dezembro de 2012. Aprova as diretrizes e normas regulamentadoras de pesquisas envolvendo seres humanos. Brasília: Ministério da Saúde, 2012. Disponível em:

https://bvsms.saude.gov.br/bvs/saudelegis/cns/2013/res0466_12_12_2012.html. Acesso em: 15 jul. 2020.

BRASIL. Portaria n. 849, de 27 de março de 2017. Inclui a Arteterapia, Ayurveda, Biodança, Dança Circular, Meditação, Musicoterapia, Naturopatia, Osteopatia, Quiropraxia, Reflexoterapia, Reiki, Shantala, Terapia Comunitária Integrativa e Yoga à Política Nacional de Práticas Integrativas e Complementares. Brasília: Ministério da Saúde, 2017. Disponível em: http://bvsms.saude.gov.br/bvs/saudelegis/gm/2017/prt0849_28_03_2017.html. Acesso em: 15 jul. 2020.

MERHY, E. E.; ONOCKO, R. Agir em saúde: um desafio para o público. São Paulo: Hucitec, 1997.

SOUZA, C. F. et al. Pré-diabetes: diagnóstico, avaliação de complicações crônicas e tratamento. Arquivos Brasileiros de Endocrinologia \& Metabologia, v. 56, n. 5, p. 275-284 jul. 2012.

\section{Como referenciar este artigo}

VASCONCELLOS DE CHAZARRETA, A.; KENJI NIHEI, O.; FERREIRA LEITE, S.; MARLON DA SILVA, C.; PEREIRA PARDINHO, H.; RIBEIRO AGUIAR, E. T.; ZAGOTTA MACHADO PINEZI, T.; KÜHL SVOBODA, W. Terapia Comunitária Integrativa como instrumento de integração, empoderamento e autocuidado de usuários de uma associação de diabéticos. Temas em Educ. e Saúde, Araraquara, v. 16, n. esp. 1, p. 327-340, set., 2020. e-ISSN 2526-3471. DOI: https://doi.org/10.26673/tes.v16iesp.1.14315

Submetido em: 20/05/2020

Revisões requeridas: $30 / 05 / 2020$

Aprovado em: 25/08/2020

Publicado em: 30/09/2020 